\title{
DERECHO DEL MAR EN BRASIL: NUEVOS DESARROLLOS
}

\section{LAW OF THE SEA IN BRAZIL: NEW DEVELOPMENTS}

Alexandre Pereira da Silva ${ }^{1}$

\begin{abstract}
Post-Doctor en Derecho por la Schulich School of Law, Dalhousie University, Halifax, Canadá. Profesor de Derecho Internacional y Miembro del Consejo Científico del Instituto de Estudios de Asia de la Universidad Federal de Pernambuco. Actualmente es Visiting Fellow en el China Institute of Boundary and Ocean Studies de la Wuhan University, Wuhan, China.

E-mail: alexandrelpsilva@hotmail.com
\end{abstract}

Received: 2017-06-16. Accepted:2017-12-10

Resumen:Elartículo hace un análisis de lasnuevas acciones emprendidas por Brasil en lo referente al Derecho del Mar, especialmente sobre los cuatro programas desarrollados actualmente por el Estado brasileño. Primeramente, el trabajo presenta dos acontecimientos importantes relacionados al Derecho del Mar en Brasil: el episodio de la "Guerra de la Langosta" y la expansión del mar territorial para doscientas millas marinas. En seguida, el trabajo examina, en términos históricos, políticos y jurídicos, los cuatro programas mencionados: el proyecto “Amazonia Azul”, el Programa Archipiélago de São Pedro y São Paulo; el Programa de Investigaciones Científicas en la Isla de Trindade y el Programa de Prospección y Exploración de los Recursos Minerales de la Zona Internacional del Atlántico Sur y Ecuatorial. En la parte final, el estudio presenta algunas conclusiones.

Palabras clave: Derecho del Mar - Brasil - Programas - Desarrollos

Abstract: The article analyses the new actions undertaken by Brazil on the Law of the Sea issues, especially the four programs that Brazil has been developing in the last years. Firstly, the paper presents two important events involving Law of the Sea in Brazil: the "Lobster War" episode and the 200-nautical mile territorial sea extension. Afterwards

$1 \mathrm{El}$ autor desea expresar su gratitud al China Institute of Boundary and Ocean Studies de la Wuhan University por la acogida como visiting fellow (2016-2018). 
the paper looks the historical, political and legal questions of the four mentioned programs: the "Blue Amazon" Project, the São Pedro and São Paulo Archipelago Program; the Scientific Program on the Island of Trindade and the Program for Prospecting and Exploration of Mineral Resources in the Equatorial and South Atlantic International Area. In the end, the study presents some concluding remarks.

Keywords: Law of the Sea - Brazil - Programs - Developments

\section{INTRODUCCIÓN}

Brasil es un país de dimensiones continentales, con un territorio de $8.514 .877 \mathrm{~km}^{2}$ y una costa extensa direccionada para el Atlántico. La franja de tierra del litoral brasileño tiene una anchura variable, extendiéndose por aproximadamente 10.800 kilómetros a lo largo de la $\operatorname{costa}^{2}$, si se contabiliza sus concavidades naturales, y posee un área de aproximadamente $514.000 \mathrm{~km}^{2}$, a lo largo de diecisiete estados costeros. Ese extenso litoral, junto a su localización geográfica, da al país una importante posición estratégica y política. Sin embargo, esa prominente posición en el Atlántico Sur no transformó Brasil en un Estado con una clara orientación marítima. En pocos momentos de su historia jurídica y política el asunto estuvo como tema principal, excepto por dos momentos en especial: en el episodio de la "guerra de la langosta", con Francia (1962-1964), y en la ampliación del mar territorial para doscientas millas marinas (1970).

Actualmente, entre las principales actividades económicas desarrolladas a lo largo de la costa brasileña están la pesca, el turismo y la explotación de gas y petróleo en la plataforma continental, siendo esta, en términos económicos, la más importantes. De las reservas probadas de petróleo de Brasil, alrededor de 96\% están localizadas en el mar, principalmente en la costa del estado de Rio de Janeiro, que detiene $84,2 \%$ de las reservas probadas offshore y $81,2 \%$ del total. A lo largo de los últimos años, el país viene ocupando la $15^{\text {a }}$ posición en el ranking

2 La extensión de la franja costera varía enormemente en la literatura sobre el tema, de 7 mil a más de 11 mil kilómetros. Tal diferencia es debido a las distintas metodologías empleadas en el cálculo de la línea costera. El dato aquí adoptado, de 10.800 kilómetros, fue obtenido en el ámbito de los estudios sobre la representatividad de los ecosistemas costeros en el Sistema Nacional de Unidades de Conservación (SNUC), y considera los recortes y concavidades naturales de la costa brasileña. Ministério do Meio Ambiente (Gerência de Biodiversidade Aquática e Recursos Pesqueiros), Panorama da conservação dos ecossistemas costeiros e marinhos no Brasil, Brasília, MMA/SBF/GBA, 2010. 
mundial de países con las mayores reservas probadas de petróleo. ${ }^{3}$

Si en el pasado Brasil tuvo un interés limitado por el Derecho del Mar, en los últimos años él viene demostrando una nueva inflexión para el Atlántico. Algunas acciones son reflejos del renovado interés por el Derecho del Mar: la extensión de la plataforma continental más allá del límite inicial de las 200 millas marinas (Proyecto “Amazonia Azul"), el Programa Archipiélago de São Pedro y São Paulo (PROARQUIPELAGO), el Programa de Investigaciones Científicas en la Isla de Trindade (PROTRINDADE) y el Programa de Prospección y Exploración de Recursos Minerales de la Zona Internacional del Atlántico Sur y Ecuatorial (PROAREA).

De esa manera, el objetivo principal de este artículo es, en un primer momento, describir los principales hechos históricos que unen Brasil al Derecho del Mar, para, en un segundo momento, analizar los desdoblamientos actuales de la política oceánica brasileña. Conocido por ser un gigante continental, los últimos quince años vienen mostrando una nueva cara brasileña: la atlántica.

\section{BRASIL Y EL DERECHO DEL MAR}

De la independencia en 1822 a los días actuales, las reglas jurídicas sobre la delimitación de los espacios marítimos adyacente a la costa brasileña pasaron por varios cambios. A lo largo del tiempo es posible percibir una serie de avanzos y retrocesos, algunas actitudes caracterizadas por una cierta timidez, mientras otras marcadas por cierta osadía. En lo que se refiere al Derecho del Mar, la mayor parte de la historia jurídica brasileña fue dedicada a la anchura del mar territorial, en especial el tema de las 200 millas marinas durante los años 1970. Sin embargo, antes de examinar este episodio, vale la pena destacar un episodio anterior a este - que, como el primero, tuvo una grande repercusión en los medios de comunicación, traspasando los límites jurídicos y políticos, y reverberando en la sociedad -, la llamada "guerra de la langosta".

\subsection{El episodio de la "guerra de la langosta" con Francia}

\section{(1962-1964)}

Entre los años 1962 y 1964 ocurrió un serio litigio involucrando Brasil y Francia en torno a la pesca de langosta en la costa del Nordeste brasileño. Los primeros hechos de lo que se conoció como "guerra de

3 AGÊNCIA NACIONAL DO PETRÓLEO, GÁS NATURAL E BIOCOMBUSTÍVEIS. Anuário estatístico brasileiro do petróleo, gás natural e biocombustiveis 2016. Rio de Janeiro: ANP, 2016, p. 71-74. 
la langosta" ocurrieron cuando un barco langostero francés, llamado Cassiopés, fue detenido en el litoral del Nordeste, en enero de 1962. La controversia surgió porque el Gobierno de Brasil negó la permisión de la pesca o captura del crustáceo en el litoral brasileño - un espacio que comenzaría en la ciudad de Recife y se extendería hacia Fortaleza - por langosteros franceses.

Desde 1961, los franceses ya mostraban un interés en el potencial de explotación de langosta en la costa brasileña, cuando, en este año, solicitó la autorización para que dos barcos franceses hicieran investigaciones sobre los recursos langosteros del Nordeste. El pedido francés fue aceptado por las autoridades brasileñas, que autorizaron la pesca por barcos franceses con la condición de que hubiera a bordo controladores de pesca de la Marina de Brasil, que verificarían los métodos de pesca y serían informados sobre los resultados de las investigaciones - tal condición fue acepta sin restricciones por la embajada francesa en Brasil. ${ }^{4}$

Con el fin de las investigaciones, el Departamento Político del Ministerio de Relaciones Exteriores (Itamaraty) advirtió al embajador de Francia que, siendo la langosta recurso de grande importancia para el Nordeste, y cuya existencia estaría amenazada por la explotación intensiva con métodos predatorios, el Gobierno brasileño no estaría dispuesto a permitir esa actividad a pescadores extranjeros. La preocupación del Gobierno de Brasil era más que razonable, una vez que durante toda la década de 1950, la pesca predatoria fue realizada en la región del Atlántico norte del continente africano, sobre todo en las costas de Senegal, Guinea y Mauritania, dónde actuaba grande parte de la frota especializada de Francia, conduciendo al agotamiento de los bancos langosteros de la región y, por lo tanto, buscando nuevas áreas de explotación, como en el caso concreto, en la costa brasileña. ${ }^{5}$

Los franceses decidieron desconsiderar las manifestaciones de las autoridades brasileñas, conduciendo sus barcos de pesca para la costa brasileña, cuando entonces fueron apresados por la Marina brasileña y luego liberados. Mientras tanto, hubo intentos de resolver el conflicto por medio de negociaciones bilaterales. Francia propuso que el asunto fuera sometido a una instancia arbitral, la Corte Permanente de Arbitraje, mientras el Itamaraty rechazó tal propuesta, pero aceptó la idea de llegar a una fórmula de modus vivendi, a la cual, sin comprender las posiciones jurídicas antagónicas de los dos países, se disciplinara el asunto creando un entendimiento entre particulares brasileños y franceses. ${ }^{6}$

4 LESSA, Antonio Carlos. A Guerra da Lagosta e outras guerras: conflito e cooperação nas relações França-Brasil (1960-1964). Cena Internacional, n. 1, 1999, p. 111-112.

5 Ibidem, p. 112.

6 Ibidem, p. 113. 
En medio a las tratativas para solucionar la controversia, en enero de 1963, las autoridades francesas comunicaron, de modo sorprendente, que dos langosteros franceses habían partido rumbo al Nordeste brasileño y que fueron nuevamente detenidos por la Marina de Guerra. Debido a la apelación del Gobierno francés, Brasil aceptó nuevamente liberarlos como un gesto de última concesión. El mes siguiente, Francia una vez más solicitó la permisión para la operación de seis langosteros en la región cuando ya estaban en las proximidades de la costa brasileña. Brasil comunicó oficialmente el impedimento de la presencia de la pesca de langostas por embarcaciones extranjeras. ${ }^{7}$

En este momento, la tensión alrededor de la "guerra de la langosta" alcanzó su punto máximo cuando el Gobierno francés decidió reaccionar de manera enérgica al comunicado brasileño, enviando el destructor Tartu para proteger las actividades de sus langosteros en el Nordeste brasileño. La prensa brasileña ha llamado a esta acción de "belicosa". En los meses siguientes, la tensión entre los gobiernos de Francia y Brasil dio lugar a la retirada de los embajadores y a la cancelación de viajes oficiales ${ }^{8}$. No obstante, no fueron registradas actitudes de animosidad entre los navíos de guerra brasileños y el destructor francés, y el asunto fue enfriando gradualmente. ${ }^{9}$

Para Raymundo de Castro, el grande mérito de la posición sostenida por Brasil en el desarrollo de este litigio consistió en la transferencia de la cuestión del campo político para el biológico. Desde el principio, el Gobierno brasileño fundamentó su posición de que la langosta es un recurso vivo del lecho de la plataforma continental, de modo que su pesca solo puede ser hecha por extranjeros con la autorización o consentimiento expreso del Estado costero. ${ }^{10}$

Los franceses se opusieron a la tesis brasileña, argumentando que la langosta es una especie nadadora y no sedentaria o bentónica. Las autoridades brasileñas respondieron, alegando que la langosta vive en contacto físico constante con el lecho del mar y que el hecho de ella dar pequeños saltos en su marcha sobre el lecho de la plataforma no era razón suficiente para que fuera considerada una especie pelágica o nadadora.

7 Ibidem, p. 114.

8 AZZAM, Issam. The dispute between France and Brazil over Lobster Fishing in the Atlantic. International Comparative Law Quarterly, vol. 13, n. 4, 1964, p. 1454.

9 MUNIZ, Tulio de Souza. O ouro do mar: o surgimento da indústria da pesca da lagosta no Brasil à condição do pescador artesanal na História do tempo presente (1955-2000). Uma narrativa sócio-histórico marítima. Fortaleza: Universidade Federal do Ceará, Dissertação (Mestrado em História), 2005, p. 52.

10 CASTRO, Raymundo Nonnato Loyola de. Aspectos fundamentais da doutrina brasileira sobre plataforma continental. Revista Brasileira de Politica Internacional, n. 47/48, 1969, p. 36. 
Por fin, recuerda Raymundo de Castro: “echamos mano de un argumento que fue decisivo: se la langosta fuera considerada especie nadadora solo porque da saltos en el lecho del mar, entonces también deberíamos incluir el canguro que da saltos en tierra firme entre las especies que vuelan". ${ }^{11}$

La "guerra de la langosta" tuvo como contribución más importante llevar para la opinión pública brasileña el debate que trata de los aspectos esenciales sobre la utilización de los recursos del mar, en especial de la plataforma continental. La repercusión del asunto en la opinión pública nacional fue expresiva, un momento raro en el que el Derecho del Mar llegó a los titulares de los periódicos.

\subsection{El mar territorial brasileño: la regla del cañón a las 200 millas marinas}

El segundo hecho jurídico y político relacionado con el Derecho del Mar que transcendió la esfera más restricta de los expertos en el tema y se tornó un interés de la opinión pública en general fue cuando Brasil, por medio del Decreto-ley n. 1.096, de 25 de marzo de 1970, expandió su mar territorial para 200 millas marinas. Antes de ser analizado el contexto en el cual ocurrió la adopción de esta acción, es importante exponer como fue la evolución del mar territorial brasileño hasta aquel momento.

Como parte del legado portugués, Brasil mantuvo la regla del cañonazo, que fue mencionada en la Circular n. 92, de 1 de julio de 1850, firmada por el Ministro de la Marina y enderezada a los presidentes de las provincias ${ }^{12}$. Como recuerda Marotta Rangel, Brasil "adhirió a la independencia con una regla distinta de aquella practicada por los otros países americanos que obtuvieron su independencia de España o Inglaterra y para quien la regla de las seis o tres millas marinas prevaleció hasta por lo menos desde la independencia". ${ }^{13}$

La regla solamente fue modificada al comienzo de la Primera

11 Ibidem, pp. 35-36. Traducción del original: “[...] lançamos, a seguir, mão de um argumento que foi decisivo: se a lagosta fosse considerada espécie nadadora só porque dá saltos no leito do mar, então deveríamos também incluir o canguru que dá saltos em terra firme entre as espécies voadoras!".

12 BEVILÁQUA, Clovis. Direito Público Internacional: a synthese dos princípios e contribuição do Brazil. Rio de Janeiro: Francisco Alves, 1910, p. 321-322.

13 RANGEL, Vicente Marotta. Brazil. In: ZACKLIN, Ralph (ed.). The changing law of the sea: Western hemisphere perspectives. Leiden: Sijthoff, 1974, p. 136. Traducción del original: “[...] acceded to independence with a rule different from that in force in other American countries which had gained independence from Spain or England and for whom the rule of six or three nautical miles prevailed, at least since independence". 
Guerra Mundial, con fines de basar la posición de neutralidad brasileña, cuando el Ministerio de Relaciones Exteriores adoptó la regla de las tres millas marinas por las Circulares n. 42 y 43, de 25 de agosto de 1914, respectivamente encaminadas a las Legaciones extranjeras y a los gobernadores y presidentes de los Estados que componían la Unión, bien como a los Ministerios de Guerra y de la Marina.

Para la Conferencia de La Haya de 1930 sobre la Codificación del Derecho Internacional, las instrucciones dadas a la delegación brasileña eran para advocar el alargamiento del mar territorial. La delegación brasileña era favorable a un mar territorial de seis millas y contraria a la institución de una zona contigua. Sin embargo, la Conferencia de La Haya de 1930 no consiguió lograr un consenso sobre la anchura del mar territorial, marcando, de esa forma, el fracaso de la Conferencia y del Proyecto Schücking ${ }^{14}$. Internamente, las instrucciones a la delegación brasileña no tuvieron impacto legislativo y el país mantuvo la anchura del mar territorial en apenas tres millas marinas. ${ }^{15}$

A pesar de la posición contraria a una zona contigua al mar territorial, pocos años después el gobierno creó una "zona de pesca costera”. Según el artículo $3^{\circ}$, parágrafo $2^{\circ}$, del Decreto n. 23. 672, de 2 de enero de 1934, que aprobó el Código de Caza y Pesca: "la pesca costera es la realizada de la costa a una distancia de 12 millas, contando para fuera".

Durante la Segunda Guerra Mundial - antes de la relación directa con el conflicto -, Brasil apoyó iniciativas regionales para la constitución de una zona de seguridad de 12 millas marinas. Con todo, el mar territorial seguía con la anchura de tres millas según lo estipulado desde el comienzo del siglo XX.

Para Marotta Rangel, la regla de las tres millas para el mar territorial fue siendo gradualmente despreciada por aquello que el cuerpo diplomático apoyaba en los encuentros internacionales ${ }^{16}$. Los "Principios de México sobre el régimen jurídico del mar", aprobado por el Consejo Interamericano de Juristas de 1956, no solo declaraba que la regla de las tres millas era insuficiente como límite del mar territorial y, por lo tanto, no era más acepta como regla general de Derecho Internacional, como también apoyaba el derecho de cada Estado establecer la anchura de su mar territorial dentro de los límites razonables.

En 1965, después que las dos primeras conferencias de las Naciones Unidas sobre el Derecho del Mar, realizadas en Ginebra

14 O'CONNELL, D. P. The International Law of the Sea. Oxford: Oxford University Press, 1982, p. 3.

15 PEREIRADA SILVA, Alexandre. O Brasil e o Direito Internacional do Mar Contemporâneo: novas oportunidades e desafios. São Paulo: Almedina, 2015, p. 227.

16 RANGEL, op. cit., p. 137. 
en 1958 y 1960, no lograron un acuerdo sobre la anchura del mar territorial, el Comité Jurídico Interamericano recomendó, con el apoyo de su presidente, el jurista brasileño Raul Fernandes, que todo Estado americano "tiene el derecho de establecer la anchura de su mar territorial hasta el límite de doce millas contadas desde la línea de base". ${ }^{17}$

Brasil solamente aumentó el mar territorial para seis millas por medio del Decreto-ley n. 44, de 18 de noviembre de 1966. Además de eso, el decreto estableció también una franja adicional, hasta la distancia de doce millas del litoral, en lo que se refiere a la prevención y a la represión de las infracciones con relación a la policía fiscal, aduanera, sanitaria o de inmigración.

Ese período, sin embargo, no iba a durar mucho. Ya en 25 de abril de 1969, el gobierno brasileño editó el Decreto-ley n. 553, alterando de nuevo los límites del mar territorial brasileño, que pasó a ser de doce millas marinas. El período en cual el mar territorial brasileño tuvo el límite de doce millas marinas sería aún más breve que el anterior. Menos de un año después, en 25 de marzo de 1970, por el Decreto-ley n. 1.098, el gobierno brasileño determinó que el límite del mar territorial pasaría a ser de 200 millas marinas. ${ }^{18}$

Las razones que llevaron Brasil a expandir su mar territorial de 12 para 200 millas marinas, en un espacio de menos de un año, son varias, de naturaleza externa e interna. En aquel particular momento histórico, había la percepción de la viabilidad de la idea por parte de las autoridades brasileñas, que recomendaban y justificaban la acción de reivindicación unilateral sobre una extensa área del mar adyacente a las costas del país. Para Araújo Castro, el impacto positivo interno de la expansión del mar territorial para 200 millas marinas, en un momento particularmente sensible para el Gobierno, fue considerado para la adopción de la medida. ${ }^{19}$

En América Latina - donde la mayoría de los países ya habían adoptado el mar territorial de 200 millas marinas - la aceptación del acto brasileño fue la mejor posible. A fin de cuentas, la posición sería reforzada con la adhesión de un miembro importante como Brasil. Entre los demás Estado en desarrollo, la medida también fue bien recibida, aunque no

17 Apud RANGEL, op. cit., p. 138.

18 PEREIRA DA SILVA, Alexandre. O novo pleito brasileiro no mar: a plataforma continental estendida e o Projeto Amazônia Azul. Revista Brasileira de Política Internacional, vol. 56, n. 1, 2013, p. 106-107.

19 ARAÚJO CASTRO, Luiz Augusto de. O Brasil e o novo Direito do Mar: mar territorial e zona econômica exclusiva. Brasília: Funag, 1989, p. 17. Además de eso, Araújo Castro aún indica que el asunto se tornó, incluso, tema de samba de João Nogueira "Esse mar é meu": "Ese mar es mío/lleve su barco para lejos de ese mar/vaya a lanzar su red/de las doscientas para allá/ pescador de ojos verdes/vaya a pescar en otro lugar". 
efusivamente como entre los latinoamericanos. ${ }^{20}$

En este momento histórico, la discusión acerca de un mar territorial de 200 millas marinas era un tema polémico y rechazado por los Estados desarrollados. Durante los trabajos de la III Conferencia de las Naciones Unidas sobre el Derecho del Mar (1973-1982), fue adoptada la solución conciliatoria: el mar territorial con anchura máxima de 12 millas marinas contadas desde las líneas de base, y se creó el concepto de zona económica exclusiva (ZEE), con un máximo de 200 millas marinas de las líneas de base, o sea, 188 millas marinas contadas desde el límite exterior del mar territorial para los Estados costeros que establecieran un mar territorial de 12 millas.

La posibilidad de una disminución en el mar territorial de 200 millas para 12 millas marinas, ya esbozada al comienzo de la III Conferencia, inicialmente fue vista con resistencia por el Gobierno de Brasil que, sin embargo, prometía no hacer oposición al consenso que se formaba sobre el tema. La probabilidad, casi una certeza, de que Brasil iba a reducir el mar territorial de 200 millas para 12 millas, en razón del proceso de negocio en curso en la III Conferencia, fue expuesta por el Ministro de Relaciones Exteriores Ramiro Saraiva Guerreiro en una entrevista para el periódico Gazeta Mercantil, en 1980. Inicialmente, la manifestación de Saraiva Guerreiro fue vista con resistencia en el Congreso Nacional.

La aprobación del texto final de la Convención de las Naciones Unidas sobre el Derecho del Mar (CONVEMAR) ocurrió el 10 de diciembre de 1982, pero el comienzo de su vigencia internacional solo iba a ocurrir doce años después, el 16 de noviembre de 1994. Con todo, Brasil ya estaba se adecuando a los nuevos parámetros establecidos en la CONVEMAR, con la aprobación de la Ley n. 8.617, de 4 de enero de 1993, anulando el Decreto-ley n. 1.098/70. Bajo esta ley, el mar territorial brasileño comprende una franja de doce millas marinas medidas desde la línea de bajamar.

La Ley n. 8.617/93 está en vigor hasta hoy en Brasil, sin alteraciones, bien como la CONVEMAR que Brasil firmó en 10 de diciembre de 1982, al fin de la III Conferencia, depositando su instrumento de ratificación el 22 de diciembre de 1988.

Sin embargo, la entrada en vigor interna de la CONVEMAR fue poco usual. Para efectos internos y siguiendo una costumbre de larga fecha consagrada, la CONVEMAR fue promulgada por medio del Decreto $\mathrm{n}$. 99.165, de 12 de marzo de 1990, que dio poder de ejecución para efectos internos de la convención. Lo que ocurre es que la CONVEMAR todavía no había entrado en vigor en el plan internacional. De esta manera, el Decreto n. 99.165 fue anulado integralmente por el Decreto n. 99.263, de 24 de mayo de 1990. En el preámbulo de este Decreto, con fines de

20 RODRIGUES, Carlos Calero. O problema do mar territorial. Revista Brasileira de Política Internacional, n. 49/50, 1970, p. 124-125. 
justificar la anulación del decreto poco más de un mes después de su expedición, consta que eso se sucedió "considerando que el Decreto n. 99.165, de 12 de marzo de 1990, abre una brecha legislativa con relación a los espacios marítimos brasileños". ${ }^{21}$

Por lo tanto, se esperó que la CONVEMAR entrara en vigor, lo que ocurrió el 16 de noviembre de 1994, para que fuera expedido un nuevo decreto de ejecutoriedad, el Decreto n. 1.530, de 22 de junio de 1995, dando efectos internos a CONVEMAR a partir de la fecha en vigor internacional.

\section{BRASIL Y UNA NUEVA MIRADA PARA EL ATLÁNTICO}

Después de la polémica acerca del mar territorial de 200 millas marinas, pacificada con la adopción de la CONVEMAR, el Derecho del Mar se retiró de los noticiarios cotidianos y volvió al debate solamente entre los expertos en Brasil. Sin embargo, el tema viene resurgiendo en los últimos años estimulado por el plan de creación de una "Amazonia Azul" y de una mayor presencia en el Atlántico Sur.

\subsection{La expansión de la plataforma continental y el proyecto}

\section{"Amazonia Azul"}

Una de las principales novedades de la CONVEMAR fue la nueva definición jurídica de la plataforma continental. En los términos de la Convención sobre la Plataforma Continental de 1958, esta era definida como "el lecho del mar y el subsuelo de las zonas submarinas [...] hasta una profundidad de 200 metros o, más allá de este límite, hasta donde la profundidad de las aguas suprayacentes permita la explotación de los recursos naturales de dichas zonas" (artículo $1^{\circ}$ ).

La definición de la plataforma continental consagrada en esta Convención no fue exclusivamente basada en características geológicas o geomorfológicas. Además de eso, se trataba claramente de un sistema sin precisión, complicado y virtualmente impracticable, una vez que implicaba, de un lado, una profundidad específica de las aguas (200 metros) y, del otro, un criterio indefinido de aprovechamiento.

Ya la definición de plataforma continental consagrada en el artículo 76 de la CONVEMAR tampoco es basada de modo exclusivo en las características geológicas o geomorfológicas, pero se utiliza de otros dos criterios para definir plataforma continental. En los términos del artículo 76.1: "la plataforma continental de un Estado ribereño comprende el lecho y el subsuelo de las áreas submarinas [...] a todo lo

21 Traducción del original: "[...] considerando que o Decreto $n^{\circ}$ 99.165, de 12 de março de 1990, abre lacuna legislativa com relação aos espaços marítimos brasileiros". 
largo de la prolongación natural de su territorio hasta el borde exterior del margen continental, o bien hasta una distancia de 200 millas marinas". Esto es, en este artículo 76.1, la CONVEMAR utilizó dos criterios para fijar los límites de la plataforma continental, el criterio geomorfológico (margen continental) y el criterio de distancia (200 millas marinas). Como resume muy bien Robert Smith y George Taft, el artículo 76.1 "define la plataforma continental de un modo que es científicamente basada, legalmente defensable y políticamente aceptable". ${ }^{22}$

De esta manera, el Estado costero tiene dos opciones: i) hincar su plataforma continental hasta una distancia máxima de 200 millas marinas, contadas desde las líneas de base a partir de las cuales se mide la anchura del mar territorial, independientemente de los factores geológicos o geomorfológicos de la misma; ii) podrá extender su plataforma continental hasta el borde exterior del margen continental, hasta una distancia que no exceda 350 millas marinas o de 100 millas marinas contadas desde la isobata de 2.500 metros, que es una línea que une profundidades de 2.500 metros.

En la hipótesis del Estado costero utilizar el segundo criterio, o sea, expandir su plataforma continental más allá del límite inicial de las 200 millas marinas, el Estado no hará por la adopción de un simple acto unilateral, pero deberá encaminar una propuesta de expansión a la Comisión de Límites de la Plataforma Continental (de ahora en delante CLPC o Comisión), un órgano técnico creado por la CONVEMAR para analizar los pleitos estatales.

Brasil fue el segundo Estado a presentar una propuesta a CLPC, el mayo de 2004 (CLCS/42) ${ }^{23}$. El pleito encaminado por el Estado brasileños es resultado del Plan de Levantamiento de la Plataforma Continental Brasileña (LEPLAC) y fue desarrollado a lo largo de dieciocho años (1986-2004), por la Junta de Hidrografía y Navegación de la Marina y contó con el apoyo técnico y científico de la Petrobras, empresa estatal petrolera. ${ }^{24}$

La propuesta brasileña de extensión de su plataforma continental para más allá de las 200 millas marinas previó una expansión de $911.847 \mathrm{~km}^{2}$. En seguida, el febrero de 2006, Brasil también hizo una adición, haciendo con que el área total se quedara con $953.525 \mathrm{~km}^{2}$. Esa área se distribuye principalmente en las regiones Norte (región del

22 Robert Smith; George Taft, "Legal Aspects of the continental shelf", in Peter J. Cook; Chris Carleton, Continental Shelf Limits: the scientific and legal interface, Oxford, Oxford University Press, 2000, p. 17. Traducción del original: "defines the continental shelf in a manner which is scientifically based, legally defensible, and politically acceptable".

23 CLCS/42, 14 September 2004. Statement by the Chairman of the Commission on the Limits of the Continental Shelf on the progress of work in the Commission.

24 VIDIGAL, Armando Amorim. Amazônia Azul: o mar que nos pertence. Rio de Janeiro: Record, 2006, p. 51. 
Cono del Amazonas y la Cadena Norte Brasileña), Sudeste (región de la Cadena Vitória-Trindade y la Meseta de São Paulo) y Sur (región de la Meseta de Santa Catarina y Cono do Rio Grande). En esos términos, el área oceánica brasileña totalizaría $4.400 .000 \mathrm{~km}^{2}$, correspondiendo, aproximadamente, a la mitad del área terrestre, lo que le equivaldría el nombre de "Amazonia Azul". ${ }^{25}$

Tras analizar los documentos de la propuesta encaminada por Brasil, la Comisión - por medio del documento CLCS/54, de 27 de abril de 2007 - no aprobó integralmente el pleito brasileño. Del total de la zona reivindicada por Brasil, la CLPC no concordó con cerca de $190.000 \mathrm{~km}^{2}$, o sea, 20\% del área extendida allá de las 200 millas marinas. ${ }^{26}$

Con todo, el Anexo II de la CONVEMAR - que trata de la Comisión de Límites de la Plataforma Continental - permite que el Estado costero, al discordar de las recomendaciones de la CLPC, presente "dentro de un plazo razonable una propuesta revista o una nueva propuesta" (artículo $8^{\circ}$, Anexo II).

Esa es la situación actual del pleito brasileño a una plataforma continental extendida, que, luego recibidas las recomendaciones iniciales de la CLPC, reinició los estudios con fines de encaminar una propuesta revista a la Comisión. Sin embargo, distinto de lo que ocurrió en 2004, el Gobierno brasileño envió a la Comisión, el 10 de abril de 2015, una sumisión parcial revista, solamente acerca del "Área Sur". Las demás regiones de la sumisión brasileña que no fueron íntegramente aceptadas por la CLPC, en especial la cadena Vitória-Trindade y meseta de São Paulo, serán sometidas a pleitos posteriores. ${ }^{27}$

Con todo, en medio a eso, la Comisión Interministerial para los Recursos del Mar (CIRM), por medio de la Resolución $\mathrm{n}^{\mathrm{o}} 3$, de 26 de agosto de 2010, admitió la propuesta de la Subcomisión para el LEPLAC, que deliberó sobre el derecho del Estado brasileño de evaluar de manera previa los pedidos de autorización para la realización de investigaciones en la plataforma continental brasileña más allá de las 200 millas marinas, determinando así que

\section{[...] independientemente del limite exterior de la Plataforma Continental (PC) más allá de las}

25 PEREIRA DA SILVA, op. cit., p. 115.

26 CLCS/54, 27 April 2007. Statement by the Chairman of the Commission on the Limits of the Continental Shelf on the progress of work in the Commission.

27 Commission on the Limits of the Continental Shelf (CLCS), Outer limits of the continental shelf beyond 200 nautical miles from the baselines: submissions to the Commission: Partial revised Submission by Brazil, Disponible en: <http://www. un.org/depts/los/clcs_new/submissions_files/submission_bra_rev.htm>. Acceso en: 16 de junio de 2017. 
200 millas marinas no haber sido definitivamente establecido, Brasil tiene el derecho de evaluar previamente los pedidos de autorización para la realización de investigación en suya PC más allá de las 200 millas marinas, teniendo como base la propuesta de límite exterior, encaminada a la Comisión de Límites de la Plataforma Continental (CLPC), en 2004. ${ }^{28}$

De ese modo, el Gobierno brasileño, por acto unilateral, mientras aguarda la posición final de la CLPC, decidió que el propio Brasil es aquel que tiene el derecho de evaluar previamente los pedidos de autorización para la realización de investigaciones en la plataforma continental brasileña extendida. La actitud brasileña es perfectamente defensable, protegiendo sus derechos mientras aguarda una nueva manifestación de la Comisión. ${ }^{29}$

El reflejo de la importancia y del interés que el tema de la "Amazonia Azul" viene despertando en la sociedad pude ser ejemplificado por la iniciativa del Senado Federal de establecer el "Día de la Amazonia Azul" (PLS 30/2014). En junio de 2015, el Proyecto de Ley 7903/14 del Senado fue aprobado por la Comisión de Constitución y Justicia (CCJ) de la Cámara de los Diputados en carácter conclusivo. El proyecto original establecía el día 10 de diciembre para recordar la fecha; sin embargo, por enmienda propuesta en la Comisión de Cultura y aprobado por la CCJ de la Cámara de los Diputados, fue instituido - por la Ley n. 13.187, de 11 de noviembre de 2015 -, el "Día de la Amazonia Azul" el 16 de noviembre, fecha de la entrada en vigor de la CONVEMAR en 1994, y no el 10 de diciembre, fecha de la firma de la Convención, tal como previsto en el proyecto original del Senado. ${ }^{30}$

\subsection{El Programa Archipiélago de São Pedro y São Paulo}

28 Disponible en: <https://www.mar.mil.br/secirm/documentos/atas/resolucao-3-2010.pdf $>$. Acceso en: 16 de junio de 2017. Traducción del original: "[...] independentemente de o limite exterior da Plataforma Continental (PC) além das $200 \mathrm{MN}$ não ter sido definitivamente estabelecido, o Brasil tem o direito de avaliar previamente os pedidos de autorização para a realização de pesquisa na sua PC além das 200 MN, tendo como base a proposta de limite exterior encaminhada à Comissão de Limites da Plataforma Continental (CLPC), em 2004".

29 PEREIRA DA SILVA, op. cit., p. 116-117.

30 Câmara dos Deputados, Aprovada comemoração do Dia Nacional da Amazônia Azul em 16 de novembro, Disponible en: < http:/www2.camara.leg.br/camaranoticias/ noticias/MEIO-AMBIENTE/490640-APROVADA-COMEMORACAO-DO-DIANACIONAL-DA-AMAZONIA-AZUL-EM-16-DE-NOVEMBRO.html>. Acceso em: 16 de junio de 2017. 


\section{(PROARQUIPELAGO)}

Rumbo al camino para aumentar su presencia en el Atlántico Sur, Brasil lanzó un segundo plan importante, el Programa Archipiélago de São Pedro y São Paulo (PROARQUIPELAGO), creado por la Resolución n. 1 de la CIRM, de 11 de junio de 1996, que estableció también el Grupo de Trabajo Permanente para Ocupación e Investigación en el Archipiélago de São Pedro y São Paulo.

El archipiélago de São Pedro y São Paulo - anteriormente conocidos como Rocas de São Pedro y São Paulo - es compuesto por seis islas mayores, cuatro menores y otras varias puntas de rocas, presentando un área total de $17.000 \mathrm{~m}^{2}$; la distancia entre los puntos extremos es de cerca de 420 metros. Se trata de uno de los puntos más inhóspito del país, una vez que el conjunto de islas no tiene playas, vegetación y agua potable. El archipiélago se queda muy próximo de la línea del Ecuador, distante cerca de $1.110 \mathrm{~km}$ de la ciudad de Natal y $520 \mathrm{~km}$ del archipiélago de Fernando de Noronha. ${ }^{31}$

Cuando se creó el PROARQUIPELAGO, el objetivo principal del programa era la implementación de una Estación Científica permanente en aquella zona, para a partir de entonces desarrollar, de forma sistemática, investigaciones científicas en el local, ocupándole de forma definitiva.

El renovado interés por São Pedro y São Paulo se dio a partir de la ratificación por Brasil de la CONVEMAR, en diciembre de 1988, en especial, en razón de lo que dispone el artículo 121:

1. Una isla es una extensión natural de tierra, rodeada de agua, que se encuentra sobre el nivel de ésta en pleamar. 2. Salvo lo dispuesto en el párrafo 3 , el mar territorial, la zona contigua, la zona económica exclusiva y la plataforma continental de una isla serán determinados de conformidad con las disposiciones de esta Convención aplicables a otras extensiones terrestres. 3. Las rocas no aptas para mantener habitación humana o vida económica propia no tendrán zona económica exclusiva ni plataforma continental

Debido a ese dispositivo, una isla es una formación natural de tierra, rodeada de agua, que posee, consecuentemente, mar territorial, zona contigua, zona económica exclusiva y plataforma continental, esto es, sus límites pueden ser extendidos hasta 200 millas marinas a

31 VIANA, Danielle de Lima. O Arquipélago de São Pedro e São Paulo: 10 anos de Estação Científica. Brasília: SECIRM, 2009, p. 15-16. 
partir de las líneas de base. Sin embargo, el párrafo $3^{\circ}$ de ese mismo artículo 121 dispone que: "las rocas que, por si propias, no sirven para la habitación humana o para la vida económica no deben tener zona económica exclusiva ni tampoco plataforma continental", esto es, las rocas tendrían apenas mar territorial (12 millas marinas) y zona contigua (otras 12 millas marinas).

Es interesante recordar que las rocas (o islas) de São Pedro y São Paulo siempre fueron llamadas de "rocas". La propia Resolución n. 1/96 de la CIRM indicó la importancia de la alteración de tal denominación cuando propuso "que sean tomadas las medidas legales necesarias para la alteración de la toponimia de las Rocas de São Pedro y São Paulo para Archipiélago de São Pedro y São Paulo".

Después de algunos estudios sobre la ocupación del archipiélago, fue presentado un nuevo cuadro toponímico para la región. Oficialmente, de acuerdo con la Carta Náutica n. 11 (1998) trazada pela Diretoria de Hidrografia e Navegação, el archipiélago de São Pedro y São Paulo es formado por un conjunto de cinco islotes (Barão de Tefé, Belmonte, São Pedro, São Paulo y Sirius) y cinco rocas (Atobá, Viuvinha, Graça Aranha, Coutinho y Sacadura Cabral).

La consecuencia más importante es que el hecho de transformarse en un archipiélago, esto es, un conjunto de islas, permite que Brasil añada $450.000 \mathrm{~km}^{2}$ de plataforma continental y zona económica exclusiva alrededor del archipiélago, área que equivale aproximadamente a 15\% de toda área marítima brasileña o $6 \%$ del territorio brasileño. ${ }^{32}$

Sin embargo, la medida no pudo simplemente limitarse a la toponimia, pues el país tuvo que ocupar efectivamente el conjunto de islotes. En 1998, ocurrió la inauguración de la Estación Científica en São Pedro y São Paulo, "a partir del cual el local permanece permanentemente habitado", según el entendimiento oficial de la CIRM. La Estación Científica, que pasó por un proceso de remodelación acabado en 2008, posee un alojamiento para cuatro personas, sala de estar, laboratorio, cocina, cuarto de baño, terraza, área para almacenamiento de agua potable y una edificación de apoyo para el generador de emergencia. ${ }^{33}$

Por lo tanto, el PROARQUIPELAGO está inserido en este contexto, que es lo de ocupar y justificar una zona económica exclusiva y una plataforma continental de 200 millas marinas en la región. Entre las competencias del programa está la de conducir un programa continuo de investigaciones científicas en la región, en las áreas de geología, geofísica, biología, recursos de pesca, oceanografía, meteorología y sismología. ${ }^{34}$

32 Ibidem, p. 11.

33 PROARQUIPELAGO, Disponible en: < https://www.mar.mil.br/secirm/portugues/ arquipelago.html>. Acceso en: 16 de junio de 2017.

34 ARRAIS, Raimundo Pereira Alencar. O nascimento de um arquipélago: São Pedro e São 


\subsection{EI Programa de Investigaciones Científicas en la Isla de la Trindade (PROTRINDADE)}

La isla de Trindade se encuentra a poco más de $1.160 \mathrm{~km}$ de Vitória, capital del estado de Espírito Santo. Posee una extensión de 10,2 $\mathrm{km}^{2}$ y es fuertemente accidentada, con elevaciones que atingen hasta 600 metros (Pico Deseado). Ya la isla de Martin Vaz - un conjunto menor de islas - se encuentra a unos $48 \mathrm{~km}$ a leste de Trindade. El archipiélago constituye el extremo leste de una cadena de montañas submarinas que tiene inicio cerca de la ciudad de Vitoria y emerge del fondo oceánico a más de 5.000 metros de profundidad donde existieron volcanes que formaron las dos islas principales, además de islas menores y rocas. Debido a su origen volcánica, hay considerable presencia de lavas, cenizas y arenas volcánicas. La última erupción volcánica ocurrió hace casi 50.000 años. $^{35}$

La posesión pacífica de la isla de Trindade que nunca fue objeto de contestación y entendida como parte integrante del territorio brasileño desde la independencia fue, en enero de 1895, ocupada por la Gran Bretaña, bajo la excusa de que la isla fue abandonada. La presencia británica causó indignación popular y provocó grande reacción diplomática. La reacción del Legislativo también fue de indignación y apoyo al Ejecutivo. ${ }^{36}$

La anexión británica se había llevado a cabo de manera confidencial con el fin de preservar los intereses comerciales de las compañías de telégrafos dispuestos a poner un cable entre Gran Bretaña y el río de la Plata, pero teniendo en cuenta la larga distancia entre la Isla Ascensión y Buenos Aires ese sería demasiado largo para el éxito comercial, una alternativa podría ser dividir en cable en dos secciones, utilizando la isla de Trindade en su ruta. ${ }^{37}$

Brasil rechazó todas las iniciativas de que la cuestión fuera solucionada por arbitramento. El argumento utilizado era el de que la isla, mismo deshabitada, no estaba abandonada. El litigio fue solucionado por la mediación del rey de Portugal D. Carlos I, de manera favorable y justa para Brasil. En agosto de 1896, el encargado británico de negocios comunicó al Ministerio de Relaciones Exteriores el reconocimiento británico de la soberanía brasileña sobre la isla. Pocos días después, la

Paulo e a presença do Estado brasileiro no Oceano Atlântico. Revista Porto, n. 2, 2012, p. 66-67. 35 PROTRINDADE, Disponible en: <https://www.mar.mil.br/secirm/portugues/ trindade.html $>$. Acceso em: 16 de junio de 2017.

36 BUENO, Clodoaldo Bueno. A República e sua politica exterior (1889-1902). Brasília: Funag, 1995, p. 333.

37 MARSTON, Geoffrey. The Anglo-Brazilian Dispute over the Island of Trindade, 1985-6. British Yearbook of International Law, vol. 54, n. 1, 1984, p. 226. 
legación inglesa en Rio de Janeiro confirmó el reconocimiento y anunció que iba a retirar las señales de la ocupación. Tras ser desocupada, fue enviado el cruzador Benjamin Constant, en enero de 1897, para hacer nuevos señales de la presencia brasileña en la isla ${ }^{38}$

En virtud de la Primera Guerra Mundial, en 1916, Trindade fue ocupada con fines de impedir su utilización por navíos adversarios. En 1941 fue nuevamente ocupada militarmente para impedir que los submarinos enemigos la utilizasen como base de apoyo. En 1950, una expedición científica fue enviada para planear la ocupación permanente de la isla. En 1957, fue creado el Puesto Oceanográfico de la Isla de Trindade. ${ }^{39}$

El PROTRINDADE, siguiendo la línea de los programas anteriores, fue aprobado por la Resolución n. 3 de la CIRM, de mayo de 2007, con la finalidad principal de promover y administrar el desarrollo de investigaciones científicas en las islas de Trindade y Martin Vaz y en el área marítima adyacente.

Además de eso, el PROTRINDADE cumple con las directrices de la Política Nacional para los Recursos del Mar (PNRM), de la Política de Defensa Nacional (PDN) y de la Política de Medio Ambiente (PNMA), y tiene otros objetivos como: construir y mantener, con el consentimiento de la Marina de Brasil instalaciones para la acomodación de investigadores y realización de investigaciones científicas, juntamente a las instalaciones que ya existen en la isla de Trindade y Martin Vaz y en la zona marítima adyacente. Uno de los principales resultados del PROTRINDADE fue la construcción de la Estación Científica de la Isla de Trindade (ECIT), en diciembre de 2011, con capacidad para hospedar hasta ocho investigadores.

\subsection{EI Programa de Prospección y Exploración de Recursos}

\section{Minerales de la Zona Internacional del Atlántico Sur y}

\section{Ecuatorial (PROAREA)}

En septiembre de 2009, por medio de la Resolución n. 3 de la CIRM, fue creado el Programa de Prospección y Exploración de Recursos Minerales de la Zona Internacional del Atlántico Sur y Ecuatorial (PROAREA), es un nuevo y relevante avanzo brasileño en su renovado interés oceánico.

El PROAREA es especialmente interesante porque,

38 KÄMPF, Martin Normann. Ilha da Trindade: a ocupação britânica e o reconhecimento da soberania brasileira (1895-1896). Brasília: Funag, 2016, p. 175-189.

39 PROTRINDADE, Disponible en: <https://www.mar.mil.br/secirm/portugues/ trindade.html $>$. Acceso en: 16 de junio de 2017. 
diferentemente de lo que ocurre en los programas analizados anteriormente, su actuación no ocurre dentro de los límites nacionales, pero en la "Zona". En los términos del artículo $1^{\circ}$ de la CONVEMAR, "por 'Zona' se entiende los fondos marinos y oceánicos y su subsuelo fuera de los límites de la jurisdicción nacional", esto es, en cierto sentido, se trata de un concepto jurídico residual, una vez que comprende los espacios marinos que no están bajo el dominio de ningún Estado. La Zona y sus recursos son patrimonio común de la humanidad (artículo 136 de la CONVEMAR).

Para cuidar de la Zona y de los recursos, la CONVEMAR creó una institución con enfoque exclusivo para esta finalidad: la Autoridad Internacional de los Fondos Marinos (de ahora en delante Autoridad). La Autoridad es la organización por medio de la cual los Estados Partes administran los recursos de la Zona. Tiene sede en Kingston, capital de Jamaica.

El hecho de que la Zona sea considerada patrimonio común de la humanidad, con todo, no quiere decir que ningún tipo de actividad pueda ser ejercida dentro de su perímetro. Esto solo impide que los Estados puedan actuar de modo aislado y unilateral en esta parte de los fondos marinos. De esa forma, la internacionalización de la Zona y sus recursos implica la administración por la Autoridad.

La idea inicial de los Estados durante el proceso de negociación de la CONVEMAR era de que la propia Autoridad pudiera realizar directamente las actividades en la Zona, por medio de la Empresa, como está prescrito en el artículo 170.1 de la CONVEMAR: "La Empresa será el órgano de la Autoridad que realizará actividades en la Zona directamente en cumplimiento del apartado a) del párrafo 2 del artículo 153 , así como actividades de transporte, tratamiento y comercialización de minerales extraídos de la Zona".

La Empresa sería, entonces, una especie de órgano operacional de la Autoridad. Sin embargo, más de veinte años después de la CONVEMAR entrar en vigor, la Empresa todavía no fue creada. Eso sucedió porque el Acuerdo Relativo a la Aplicación de la Parte XI de la CONVEMAR (1994) prácticamente inviabilizó su establecimiento. El modelo vigente actualmente privilegia la exploración de la Zona vía empresas privadas que presentan planes de trabajo que deben ser patrocinados por Estados Partes de la CONVEMAR.

Hasta el mes de junio de 2017, la Autoridad había firmado 27 contratos de exploración, que cubren más de un millón de kilómetros cuadrados del fondo del mar. Diecisiete de esos contratos se refieren a la explotación de nódulos polimetálicos, seis de sulfuros polimetálicos y cuatro de cortezas de ferromanganeso de cobalto. ${ }^{40}$

40 ISA. Deep Seabed Minerals Contractors. Disponible en: <https://www.isa.org.jm/deepseabed-minerals-contractors $>$. Acceso en: 16 de junio de 2017. 
Según el sitio electrónico del PROAREA, los objetivos del programa, entre otros son: i) la ampliación de la presencia brasileña en el Atlántico Sur y Ecuatorial; ii) la recolección de datos para dar soporte a futuras requisiciones de áreas de prospección y exploración mineral junto a la Autoridad Internacional de los Fondos Marinos; e, iii) la obtención de informaciones técnicas, económicas y ambientales necesarias para que empresas, públicas y privadas, y órganos gubernamentales puedan desarrollar actividades de explotación mineral y gestión ambiental en el área allá de la jurisdicción brasileña. Y entre las principales actividades de trabajo del PROAREA están: i) integración y sistematización de informaciones; ii) evaluación de la potencialidad mineral; iii) estudios de viabilidad económica, técnica, ambiental y legal; e, iv) prospección y exploración de recursos minerales (contrato con la Autoridad). ${ }^{41}$

Sobre ese último punto, vale la pena señalar que Brasil ya presentó su plan de trabajo en la Zona para la Autoridad. La propuesta brasileña fue encaminada en diciembre de 2013 para la exploración de cortezas de ferromanganeso de cobalto (cobalt-rich ferromanganese crusts) en la Zona, por medio de la Companhia de Pesquisa de Recursos Minerales (CPRM), que es una compañía estatal.

La zona que figura en el plan de trabajo de la CPRM es en la región del Elevado do Rio Grande (Rio Grande Rise), cerca de 1.500 $\mathrm{km}$ de la ciudad de Rio de Janeiro. El plan de trabajo fue aprobado inicialmente en la Comisión Jurídica y Técnica, y posteriormente por el Consejo de Autoridad. La zona de exploración comprende 150 bloques, cada uno con un área de $20 \mathrm{~km}^{2}$. Los bloques, de forma rectangular, están agrupados en ocho grupos, cada uno con 5 a 56 bloques contiguos. Todos los bloques se encuentran completamente dentro de una zona geográfica de un tamaño que no excede a $550 \mathrm{~km}^{2}{ }^{42}$

El contrato entre la Autoridad Internacional y la CPRM fue firmado el 9 de noviembre de 2015 y tiene duración inicial de quince años. De acuerdo con el plan de trabajo presentado por la CPRM, la ejecución será realizada en tres fases, con duración de cinco años cada una. En la primera etapa serán hechos estudios de sondeos exploratorios marinos. En la segunda etapa, será realizada una evaluación de las características mineralógicas, estructurales y geomorfológicas de la región. Y, en la tercera etapa, el plan de trabajo prevé una selección de las zonas para estudio de viabilidad económica, ambiental y técnica de

41 PROAREA, Disponible en: <https://www.mar.mil.br/secirm/portugues/proarea. html $>$. Acceso en: 16 de junio de 2017.

42 INTERNATIONAL SEABED AREA (ISA). ISBA/20/C/30, 9 July 2014. Report and recommendations of the Legal and Technical Commission to the Council of the International Seabed Authority relating to an application for the approval of a plan of work for exploration for cobalt-rich ferromanganese crusts by Companhia de Pesquisa de Recursos Minerais. 
los depósitos minerales identificados. ${ }^{43}$

\section{CONCLUSIÓN}

El creciente interés por el Atlántico por parte del Estado brasileño es significativo y variado. Significativo en el sentido de que pleitea vastas áreas de actuación, sea en el caso del proyecto "Amazonia Azul", por medio de expansión de su plataforma continental, sea en el caso del PROAREA, que pretende explorar una región que se encuentra más allá de su jurisdicción nacional. Y es una presencia variada de modo que otras iniciativas, como el PROTRINDADE y el PROARQUIPELAGO, buscan consolidar y profundizar su presencia en archipiélagos que son incontestablemente brasileños.

Por lo tanto, el Derecho del Mar, tradicionalmente relegado a una posición de poca importancia en el contexto histórico, jurídico y político nacional - excepto por los episodios de la "guerra de la langosta" y de la expansión del mar territorial para 200 millas marinas - deberá ser visto con una nueva visión estratégica nacional, marcando una mayor presencia del país en el Atlántico Sur.

La costa brasileña es utilizada de diversas formas: fuente de alimentos, escenario de actividades recreativas y deportivas, turismo, de actividades relacionadas con la explotación de petróleo y gas, entre muchas otras. El momento ahora es de fortalecimiento del Derecho del Mar en Brasil, que satisfaga ese escenario de creciente interés y presencia atlántica.

\section{REFERENCIAS}

AGÊNCIA NACIONAL DO PETRÓLEO, GÁS NATURAL E BIOCOMBUSTÍVEIS (ANP). Anuário estatístico brasileiro do petróleo, gás natural e biocombustíveis 2016. Rio de Janeiro: ANP, 2016.

ARAÚJO CASTRO, Luiz Augusto de, O Brasil e o novo direito do mar: mar territorial e zona econômica exclusiva, Brasília, Funag, 1989.

ARRAIS, Raimundo Pereira Alencar. O nascimento de um arquipélago: São Pedro e São Paulo e a presença do Estado brasileiro no Oceano Atlântico. Revista Porto, n. 2, 2012, p. 42-69.

AZZAM, Issam. The dispute between France and Brazil over Lobster Fishing in the Atlantic. International Comparative Law Quarterly, vol. 13, n. 4, 1964, p. 1453-1459.

43 Disponible en: <http://www.cprm.gov.br/publique/Noticias/Contrato-assinado-entreCPRM-e-ISBA-preve-investimento-de-11-milhoes-de-dolares-na-exploracao-do-AtlanticoSul-4113.html?from_info_index=31>.Acceso en: 16 de junio de 2017. 
BEVILÁQUA, Clóvis. Direito Público Internacional: a synthese dos princípios e contribuição do Brazil. Rio de Janeiro: Francisco Alves, 1910.

BUENO, Clodoaldo. A República e sua política exterior (1889-1902). Brasília: Funag, 1995.

CÂMARA DOS DEPUTADOS, Aprovada comemoração do Dia Nacional da Amazônia Azul em 16 de novembro, Disponible en: $\quad<$ http://www2.camara.leg.br/camaranoticias/noticias/MEIOAMBIENTE/490640-APROVADA-COMEMORACAO-DO-DIANACIONAL-DA-AMAZONIA-AZUL-EM-16-DE-NOVEMBRO. html $>$. Acceso en: 16 de junio de 2017.

CASTRO, Raymundo Nonnato Loyola de. Aspectos fundamentais da doutrina brasileira sobre plataforma continental. Revista Brasileira de Política Internacional, n. 47/48, 1969, p. 19-41.

COMMISSION ON THE LIMITS OF THE CONTINENTAL SHELF (CLCS). Outer limits of the continental shelf beyond 200 nautical miles from the baselines: submissions to the Commission: Partial revised Submission by Brazil. Disponible en: <http://www.un.org/depts/los/ clcs new/submissions files/submission bra rev.htm>. Acceso en: 16 de junio de 2017.

. CLCS/42, 14 September 2004. Statement by the Chairman of the Commission on the Limits of the Continental Shelf on the progress of work in the Commission.

. CLCS/54, 27 April 2007. Statement by the Chairman of the Commission on the Limits of the Continental Shelf on the progress of work in the Commission.

INTERNATIONAL SEABED AUTHORITY (ISA), ISBA/20/C/30, 9 July 2014. Report and recommendations of the Legal and Technical Commission to the Council of the International Seabed Authority relating to an application for the approval of a plan of work for exploration for cobalt-rich ferromanganese crusts by Companhia de Pesquisa de Recursos Minerais.

KÄMPF, Martin Normann. Ilha da Trindade: a ocupação britânica e o reconhecimento da soberania brasileira (1895-1896). Brasília: Funag, 2016.

LESSA, Antonio Carlos. A Guerra da Lagosta e outras guerras: conflito e cooperação nas relações França-Brasil (1960-1964). Cena Internacional, n. 1, 1999, p. 109-120.

MARSTON, Geoffrey. The Anglo-Brazilian Dispute over the Island of Trindade, 1985-6. British Yearbook of International Law, vol. 54, n. 1, 
1984, p. 222-239.

MINISTÉRIO DO MEIO AMBIENTE (MMA). Gerencia de Biodiversidade Aquática e Recursos Pesqueiros, Panorama da conservação dos ecossistemas costeiros e marinhos no Brasil, Brasília, $\mathrm{MMA} / \mathrm{SBF} / \mathrm{GBA}, 2010$.

MUNIZ, Túlio de Souza. O ouro do mar: do surgimento da indústria da pesca da lagosta no Brasil à condição do pescador artesanal na História do tempo presente (1955-2000). Uma narrativa sócio-histórico marítima. Fortaleza: Universidade Federal do Ceará Dissertação (Mestrado em História), 2005.

O'CONNELL, D. P. The International Law of the Sea. Oxford: Oxford University Press, 1982.

PEREIRA DA SILVA, Alexandre, O Brasil e o Direito Internacional do Mar Contemporâneo: novas oportunidades e desafios. São Paulo: Almedina, 2015.

O novo pleito brasileiro no mar: a plataforma continental estendida e o Projeto Amazônia Azul. Revista Brasileira de Política Internacional, vol. 56, n. 1, 2013, p. 104-121.

PROAREA, Disponible en: < https://www.mar.mil.br/secirm/portugues/ proarea.html>. Acceso en: 16 de junio de 2017.

PROARQUIPELAGO, Disponible en: <https://www.mar.mil.br/ secirm/portugues/arquipelago.html>. Acceso en: 16 de junio de 2017.

PROTRINDADE, Disponible en: <https:/www.mar.mil.br/secirm/ portugues/trindade.html>. Acceso en: 16 de junio de 2017.

RANGEL, Vicente Marotta. Brazil. In: ZACKLIN, Ralph (ed.). The changing law of the sea: Western hemisphere perspectives. Leiden: Sijthoff, 1974.

RODRIGUES, Carlos Calero. O problema do mar territorial. Revista Brasileira de Política Internacional, n. 49/50, 1970, p. 118-130.

SMITH, Robert; TAFT, George. Legal Aspects of the Continental Shelf. In: COOK, Peter J.; CARLETON, Chris M. Continental Shelf Limits: the scientific and legal interface. Oxford: Oxford University Press, 2000, p. 17-24.

VIANA, Danielle de Lima. O Arquipélago de São Pedro e São Paulo: 10 anos de Estação Científica. Brasília: SECIRM, 2009.

VIDIGAL, Armando Amorim. Amazônia Azul: o mar que nos pertence. Rio de Janeiro: Record, 2006. 